\title{
In silico Evaluation of Impurities Found in Some Generic Drugs of Piperacillin/ Tazobactam
}

\author{
Shigeru Fujimura ${ }^{1,2^{*}}$, Takumi Sato ${ }^{1}$ and Akira Watanabe ${ }^{2}$ \\ ${ }^{1}$ Division of Clinical Infectious Diseases \& Chemotherapy, Graduate School of Pharmacy, Tohoku Medical and Pharmaceutical University, Sendai, Japan \\ ${ }^{2}$ Research Division for Development of Anti-Infective Agents, Institute of Development, Aging and Cancer, Tohoku University, Sendai, Japan
}

"Corresponding author: Shigeru Fujimura, Division of Clinical Infectious Diseases \& Chemotherapy, Graduate School of Pharmacy, Tohoku Medical and Pharmaceutical University, 4-4-1 Komatsushima, Aoba-ku, Sendai, 981-8558, Japan, Tel: 81227270169; E-mail: sfuji@tohoku-mpu.ac.jp

Received date: March 30, 2017; Accepted date: April 13, 2017; Published date: April 20, 2017

Copyright: $\odot 2017$ Fujimura S, et al. This is an open-access article distributed under the terms of the Creative Commons Attribution License, which permits unrestricted use, distribution, and reproduction in any medium, provided the original author and source are credited.

\begin{abstract}
Characterization of impurities present in generic antimicrobial agents is not required according to the approval criteria for their sale worldwide. To assess the safety of generics, we conducted a structural estimation of impurities in six piperacillin/tazobactam generics and carried out an in silico analysis. Namely, these generics were compared with the brand name drug by HPLC and LC-MS. The mutagen that in silico analysis showed alert was detected in five of six generic products. It was estimated that this mutagen was an azido group of the precursor used in the synthesis process of tazobactam whose patent has expired. With regards to this mutagenic substance, pharmaceutical companies marketing a generic Piperacillin/Tazobactam product will have to check presence or absence of this mutagen before introducing the product to the market.
\end{abstract}

Keywords: Piperacillin/Tazobactam; Generic drug; Mutagen; In silico; Antibiotics

\section{Background}

Piperacillin/Tazobactam is a combination antibiotic containing piperacillin with strong antibacterial activity against Pseudomonas aeruginosa and the $\beta$-lactamase inhibitor tazobactam. Their blending ratio used to be $4: 1$ but was changed to 8:1 in Japan in 2008 for its use mainly in severe infections as drugs that may be given at a high dose in accordance with the Pharmacokinetics-Pharmacodynamics (PK-PD) hypothesis. Generic products of this combination became commercially available in Japan in 2015. Most of bulk powders of generic antibiotics used so far in Japan are manufactured abroad, mainly in China and India, and then imported by Japan. Some problems have been reported with these generic antibiotics $[1,2]$. We demonstrated that in many generic vancomycin injections the amount of bulk powder per vial is about $15 \%$ less than in the original drug [1]. Similarly, the content of teicoplanin was found to be lower than that in the brand drug [2]. Problems with generic products of meropenem have also been pointed out. Agudelo et al. reported the therapeutic nonequivalence of generic products with the original drug in animal models of infection [3]. In addition, since the grain size of bulk powder of all generic meropenem products was larger than that of the brand drug, the dissolution time in physiological saline was three times longer compared to the brand drug [4]. Besides since the particle size of the bulk powder of all imported meropenems is larger than that of the brand drug, this was identified as the cause of being less likely to dissolve. Furthermore, it was reported that generic Piperacillin/ Tazobactam was pharmaceutic difference and therapeutically nonequivalence [5,6]. A generic product is approved as a drug as long as its main ingredient is the same as that of the original drug. Therefore, excipients and impurities contained in the generic product are not necessarily the same as those in the original drug. The purpose of this study is to study the safety of impurities contained in the bulk powder of the generic Piperacillin/Tazobactam products used in Japan. Both piperacillin and tazobactam were developed by Japanese pharmaceutical companies and are used throughout the world. However, the bulk powder of those generic products is produced abroad, including China, India, Colombia and Slovenia.

\section{Methods}

The Piperacillin/Tazobactam used in this study was the $2.25 \mathrm{gm} / \mathrm{vial}$ from six companies chosen randomly from among the generic products marketed in Japan and the original drug, seven products in total. HPLC was performed in accordance with the 16th edition of the Japanese Pharmacopoeia [7]. In summary, when a peak different from the original drug was detected, the peak area of that substance was measured with the standard solutions of piperacillin and tazobactam using the automatic integration method. This analysis was performed three times to confirm the repeatability. For the operating condition the measurement wavelength was set at $220 \mathrm{~nm}$ and the column used was Inertsil ODS-3, $3 \mathrm{~m}, 3.9 \mathrm{~mm} \times 100 \mathrm{~mm}$. Concentration of injection test solution is $1 \mathrm{mg} / \mathrm{ml}$ as Piperacillin potency. Mobile phases A and B were dibasic potassium phosphate and acetonitrile, respectively. The liquid chromatography-mass spectrometry (LC/MS) is equipped with a $210 \mathrm{~nm}$ detector, $4.6 \mathrm{~mm} \times 50 \mathrm{~mm}$ ODS column that contains $1.8 \mu \mathrm{m}$ packing, $0.25 \mathrm{ml} / \mathrm{min}$ flow rate, $40^{\circ} \mathrm{C}$ column temperature to measure relative substances with high sensitivity. Concentration of injection test solution is $2 \mathrm{mg} / \mathrm{ml}$ as piperacillin potency. $0.1 \%$ formic acid medium/acetonitrile (92:8) are used as mobile phase, and flow rate is controlled by gradient method.

\section{Results}

Impurity A was detected at $0.04-0.05 \%$ in all generic products (Nos. 3-7), except generic No. 2 and the brand drug No. 1 (Figure 1). Impurity B was detected at $0.03-0.04 \%$ in all generic products. The molecular weight of impurities $\mathrm{A}$ and $\mathrm{B}$ was determined using LC- 
Page 2 of 3

MS/MS (Shimadzu: Tokyo). The mass range was measured from $\mathrm{m} / \mathrm{z}$ 50-1200 and the molecular weights of impurities A and B were 274 and 562 , respectively. Based on these molecular weights, the chemical structure was estimated according to Scifinder (American Chemical Society) and the Patent Publication Information, and in silico analysis was performed according to Derek Nexus (CTC Life Science: Tokyo) to predict the toxicity of each substance. The chemical structures of impurities A and B and their origin are shown in Table 1. Among them impurity A was recognized as a mutagenic substance due to the organic azide group it contained [8]. In this study we demonstrated that the mutagenic substance was contained in most generic Piperacillin/Tazobactam products excluding one company's product. According to the in silico analysis, the alerted substance was in the azide group contained in impurity $A$ and was estimated to be 4-Thia-1azabicycloheptane-2-carboxylic acid, 3-(azidomethyl)-3-methyl-7oxo-,4,4-dioxide used in the synthetic process of tazobactam (Figure $1)$.

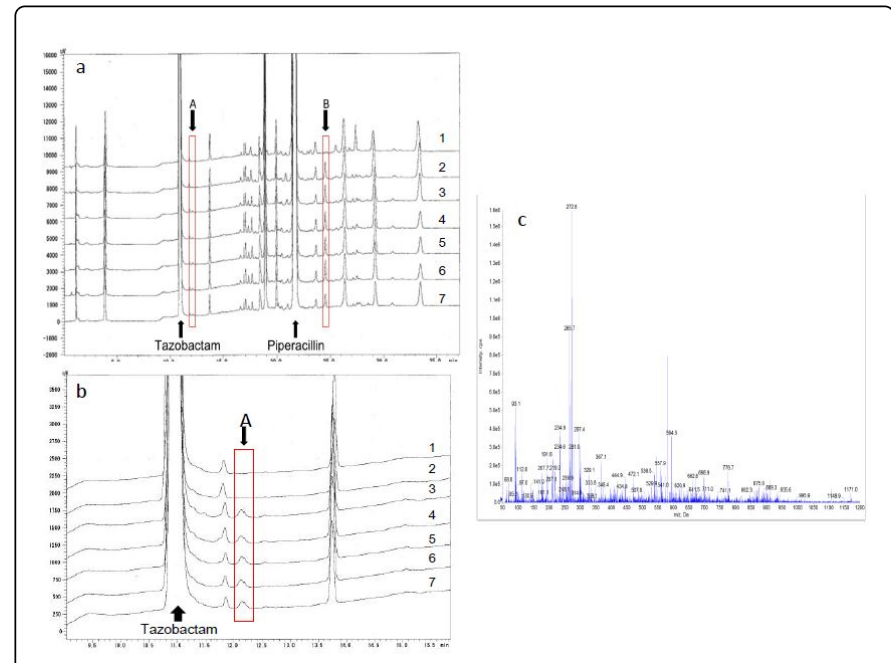

Figure 1: LC/MS scan mode (range, $\mathrm{m} / \mathrm{z} 50$ to 1,200 ) of the related substance of tazobactam extracted from generic 5 generic products (no. 3-7). (a) HPLC spectrograms of No.1 (brand) and no. 2-7 (generics); (b) enlargement of Figure a; (c) Centroids graphs describing the composition masses of related substance (arrow A peak of generic no.3). In 5 generic products (no.3-7) a peak A was detected at $12.2 \mathrm{~min}$, it had a main molecular mass of $\mathrm{m} / \mathrm{z} 273$ [M$\mathrm{H}]^{-}$that was absent in the HPLC spectra of the brand drug and generic product no. 2. The chemical structural formula (right) of this peak was estimated by in silico analysis.

\section{Discussion}

Currently, generic Piperacillin/Tazobactam products from 11 companies are commercialized in Japan. Most generic Piperacillin/ Tazobactam products had false positive galactomannan antigen values [9]. Since this trend was not common to all generic products, this indicates that there is at least one generic pharmaceutical company that purifies the imported bulk powder to remove impurities. In other words, this substance is used in the synthesis tazobactam suggesting that it is an unreacted product that failed to perform a perfect tazobactam synthetic reaction. On the other hand, the synthetic method for the brand tazobactam is different and impurity $\mathrm{A}$ (mutagenic substance) including the organic azide group is not used in the synthetic process $[10,11]$.

\begin{tabular}{|c|c|c|c|}
\hline Impurities & Analyzed compound & $\begin{array}{l}\text { Estimated structural } \\
\text { formula }\end{array}$ & Derivation \\
\hline \multirow{3}{*}{ A } & $\mathrm{CF}^{\mathrm{a}}: \mathrm{C}_{8} \mathrm{H}_{10} \mathrm{~N}_{4} \mathrm{O}_{5} \mathrm{~S}$ & & \multirow{3}{*}{ tazobactam } \\
\hline & $M W^{b}: 274$ & & \\
\hline & Content: $0.04-0.05 \%$ & & \\
\hline \multirow{3}{*}{ B } & $\mathrm{CF}^{\mathrm{a}}: \mathrm{C}_{25} \mathrm{H}_{33} \mathrm{~N}_{5} \mathrm{O}_{8} \mathrm{~S}$ & & \multirow{3}{*}{ piperacillin } \\
\hline & MW': 563 & & \\
\hline & Content: $0.03-0.04 \%$ & & \\
\hline $\mathrm{C}$ & ormula; 'b $\mathrm{MW}$ : Mo & & \\
\hline
\end{tabular}

Table 1: Chemical structure estimate of impurities by in silico analysis.

Since the international patent for the synthetic method used to produce the brand drug has not expired, companies producing generic Piperacillin/Tazobactam products are considered to have no alternative but to use the synthetic method using this mutagenic substance A. The aforementioned galactomannan antigen and mutagenic substance $\mathrm{A}$ are not detected in all generic products. Piperacillin/Tazobactam from the company from which the mutagenic substance A was not detected in this study was found to be negative to galactomannan antigen as well. As galactomannan antigen is derived from penicillin used in the biosynthetic process of piperacillin, a procedure to remove it is newly required. The brand drug manufacturer and some manufacturers of generic products carry out some processing to remove impurities. We infer that one of them probably succeeded in removing mutagenic substance A through a purification treatment of Piperacillin/ Tazobactam. Generic products have the same main ingredient as the brand drug but are known to have different additives and excipients. This study reveals that there are drugs that the patent for the synthetic method is still protected though the patent for the main ingredient expired. In other words, when a generic pharmaceutical company manufactures a drug using an outdated off-patent synthetic method, the study demonstrated that this may pose a risk for inclusion of hazardous substances such as mutagenic substances. This report indicates for the first time in the world that a mutagenic substance may be contained in generic antibiotics and we believe this is an important challenge concerning the dissemination and promotion of future generic drugs. Dissemination of generic drugs is effective in holding down soaring medical costs $[12,13]$. However, this requires the assurance of not only drug effects but also their safety $[14,15]$. In the US, EU and Japan, hazardous substances present in drugs are handled in accordance with the Guideline M7 for Assessment and control of DNA reactive (mutagenic) impurities in pharmaceuticals to limit potential carcinogenic risk as defined by the International Conference on Harmonization of Technical Requirements for Registration of Pharmaceuticals for Human Use (ICH). ICH-M7, 4.4 does not require additional action toward a marketed drug simply on account of alert demonstrated through in silico evaluation [16]. Therefore, it is considered that the results from this study alone will pose no problem 
to marketing of generic Piperacillin/Tazobactam containing mutagenic elements. However, the calculation of the acceptable intake of a mutagenic impurity depending on the treatment period and dose were shown to the ICH-M7, 7.3 [17]. Because the period of treatment with Piperacillin/Tazobactam is usually one month or less, the daily acceptable intake thus amounts to $120 \mathrm{gm}$. When the value is divided at $2 \mathrm{gm}$ that is daily maximum dose of tazobactam, allowable limit of the mutagen are $60 \mathrm{ppm}$ or less. However, the content of the mutagenic substance A detected by HPLC was $0.04-0.05 \%$ as calculated from the peak ratio, significantly exceeding the allowable limit of $60 \mathrm{ppm}$. With regards to this mutagenic substance, pharmaceutical companies marketing a generic Piperacillin/Tazobactam product must check it for the presence or absence of this mutagenic substance and perform Ames testing to confirm its safety before introducing the product to the market. The key to dissemination of generic drugs lies in the assurance of drug safety and steady supply by each generic pharmaceutical company.

\section{Conflicts of Interest}

Shigeru Fujimura and Takumi Sato has no conflicts of interest to declare. Akira Watanabe has received speaker's honorarium from MSD K.K., Kobayashi Pharmaceutical Co., Ltd., Shionogi \& Co., Ltd., Daiichi Sankyo Co., Ltd., Taisho Toyama Pharmaceutical Co., Ltd., Sumitomo Dainippon Pharma Co., Ltd., Mitsubishi Tanabe Pharma Co., Chuga Pharmaceutical Co., Ltd., Pfizer Japan Inc., UCB Japan Co. Ltd., donation from Astellas Pharma Inc., Daiichi Sankyo Co., Ltd., Sumitomo Dainippon Pharma Co., Ltd., payments for a manuscript drafting and editing from Iyaku (Medicine and Drug) Journal Co., Ltd., and grant support from Kyorin Pharmaceutical Co., Ltd., Shionogi \& Co., Ltd., Daiichi Sankyo Co., Ltd., Taisho Toyama Pharmaceutical Co., Ltd., Taiho Pharmaceutical Co., Ltd. Toyama Chemical Co., Ltd., FUJIFILM Pharma Co., Ltd. and Meiji Seika Pharma Co., Ltd.

\section{References}

1. Fujimura S, Watanabe A, Fuse K, Kikuchi T, Gomi K, et al. (2008) In vitro susceptibility of clinical isolates of methicillin-resistant Staphylococcus aureus (MRSA) to manufactured generic drugs compared with the brand vancomycin. Int J Antimicrob Agents 31: 391-392.

2. Fujimura S, Fuse K, Takane H, Nakano Y, Gomi K, et al. (2011) Antibacterial effects of brand-name teicoplanin and generic products against clinical isolates of methicillin-resistant Staphylococcus aureus. J Infec Chemother 17: 30-33.

3. Agudelo M, Rodriguez CA, Pelaez CA, Vesga O (2014) Even apparently insignificant chemical deviations among bioequivalent generic antibiotics can lead to therapeutic nonequivalence: the case of meropenem. Antimicrob Agents Chemother 58: 1005-1018.

4. Fujimura S, Watanabe A (2012) Generic antibiotics in Japan. J Infect Chemother 18: 421-427.

5. Zheng H, Truong J, Carroll F, Pai MP (2015) Do formulation differences between the reference listed drug and generic piperacillin-tazobactam impact reconstitution? Antimicrob Agents Chemother 59: 1767-1769.

6. Rodriguez CA, Agudelo M, Aguilar YA, Zuluaga A, Vesga O (2016) Impact on bacterial resistance of therapeutically nonequivalent generics: The case of piperacillin-tazobactam. PLoS One 11: e0155806.

7. The Minister of Health, Labour and Welfare (2011) Physical methods chromatography, 2.01 liquid chromatography (16th edn.) The Japanese pharmacopoeia pp: 42-45.

8. Matsumura Y, Shinozawa T, Matsushita H, Terao Y (1995) Mutagenicity of alkyl azides. Bio Pharm Bull 18: 1805-1807.

9. Demiraslan H, Atalay MA, Eren E, Demir K, Kaynar L, et al. (2016) Assessing the risk of false positive serum galactomannan among patients receiving piperacillin/tazobactam for febrile neutropenia. Med Mycol.

10. Kocyigit O, Kursunlu AN, Guler E (2010) Complexation properties and synthesis of a novel Schiff base with triphenylene nucleus. J Hazard Mat 183: 334-340.

11. Kursunlu AN, Guler E, Sevgi F, Ozkalp B (2013) Synthesis, spectroscopic characterization and antimicrobial studies of $\mathrm{Co}(\mathrm{II}), \mathrm{Ni}(\mathrm{II}), \mathrm{Cu}(\mathrm{II})$ and $\mathrm{Zn}$ (II) complexes with Schiff bases derived from 5-bromosalicylaldehyde. J Mol Struct 1048: 476-481.

12. Hakim A, Ross JS (2017) High prices for drugs with generic alternatives. The curious case of duexis. JAMA Intern Med 177: 305-306.

13. Bennett CL, Sartor OA, Armitage JO, Kantarjian H (2017) Biosimilar and generic cancer drugs unlikely to bend cost curve in the USA. Lancet Oncol 18: 22-23.

14. Gupta R, Kesselheim A, Ross JS (2017) Prioritization of generic drug review-to reply. JAMA Intern Med 177: 141-142.

15. Yang YT, Nagai S, Chen BK, Qureshi ZP, Lebby AA, et al. (2016) Generic oncology drugs: Are they all safe? Lancet Oncol 17: e493-e501.

16. International Conference on Harmonization expert working group (2014) Considerations for marketed products. ICH-M7 pp: 3-4.

17. International Conference on Harmonization expert working group (2014) 7.3 Acceptable intakes in relation to LTL exposure. ICH-M7 pp: $8-9$. 